\title{
ELEMENTOS PARA UNA DELIMITACIÓN ENTRE VETTONES Y CARPETANOS EN LA PROVINCIA DE TOLEDO
}

\author{
M. ${ }^{a}$ PILAR GONZÁLEZ-CONDE PUENTE
}

\begin{abstract}
El límite territorial entre Vettones y Carpetanos no tiene un reflejo preciso en las fuentes clásicas, por lo cual es necesario introducir en su análisis elementos de tipo epigráfico y arqueológico. Así la zona de hallazgos de esculturas de toros y verracos, presentes en el mundo vettón, pero no en el carpetano, y el uso de la onomástica, permiten sugerir una hipótesis para establecer los límites geográficos entre ambos pueblos sobre la base de sus diferencias culturales.

The territorial limit between Vettones and Carpetani has not an exact reflexion in classical sources, so it is necessary to introduce in its analisis elements of epigraphic and archaeologic kinds. So, the area of discoveries of bull and boar sculptures present in the Vettones world, but not in the Carpetani world, and the use of the onomastic, allow us to suggest hypothesis to establish the geographical limits between the two peoples, on the grounds of their cultural differences.
\end{abstract}

Dentro del problema de los límites entre las diferentes unidades étnicas peninsulares, la delimitación entre lo vettón y lo carpetano plantea una serie de dificultades, por la fragmentaria información de que disponemos. Allí donde los factores geográficos no son significativos, sólo cabe un estudio conjunto de las escasas menciones de la literatura clásica con la documentación epigráfica y arqueológica.

Las numerosas noticias de las fuentes clásicas sobre los Vettones no establecen con precisión hasta dónde llegaba su área de influencia hacia oriente, y aún son más parcas en lo que a sus vecinos Carpetanos se refiere, aunque sí recogen algunos contactos entre ambos pueblos durante la conquista romana de la Meseta (1).

(1) Una recopilación de fuentes para Carpetania en VALIENTE y BALMASEDA 1983; también GONZÁLEZCONDE 1987, 25ss.
En su parte septentrional, el límite quedaba establecido por una frontera natural montañosa, constituída por la sierra de Guadarrama y sus estribaciones, que dejan al noroeste el territorio vettón de la actual ciudad de Ávila, y al sur los valles carpetanos del Henares y Guadarrama; ambos claramente diferenciados en lo que se refiere a su identidad cultural, e incluso en su actitud ante el invasor romano, que encontró entre los Vettones una resistencia que los Carpetanos no ofrecieron, probablemente por su debilidad defensiva y por su falta de cohesión interna (GONZÁLEZ-CONDE 1987, 25ss.). En fin, se trata de una división que no iría lejana al límite provincial actual entre Ávila y Madrid.

Más al sur, la provincia actual de Toledo es el ámbito en donde se suscita la cuestión de los límites, que indudablemente dividían ese territorio entre dos zonas de influencia étnica diferenciadas: una parte occidental vettona y un sector oriental carpe- 
tano. Dado que nos encontramos en pleno valle del Tajo, no es fácil precisar hasta dónde llegaría el control de unos y otros, y la arteria de comunicación que constituye el río tampoco facilita la tarea.

Ante la imposibilidad de establecer una línea de demarcación precisa, ya con anterioridad optamos por fijar un límite provisional, que dejara al este lo que parecía corresponder al ámbito carpetano ( $i b i$ dem, 13ss.). Así, entendimos en su momento como integrados en Carpetania varios lugares, con hallazgos arqueológicos o epigráficos, situados entre Toledo y Talavera de la Reina, y que constituirían el extremo occidental de Carpetania. Parece necesaria una argumentación más precisa para justificar esa división, especialmente considerando el problema de la adscripción de Caesarobriga.

En primer lugar, las fuentes clásicas proporcionan algunos datos significativos, aunque no determinantes si se analizan de forma aislada. Sabemos por Plinio que los caesarobrigenses fueron integrados por los romanos en la provincia de Lusitania, en calidad de stipendiarii (Plinio, Nh. IV, 118), mientras Toletum quedaba en la citerior. Este dato queda además corroborado por la existencia de una mujer caesarobrigense, Domitia Proculina, que fue flaminica provincial en Lusitania, tras haberlo sido en su propio municipio (CIL II 895; ETIENNE 1974r., 166, 167, 239). Con todo ello se plantea el problema, por otra parte bastante debatido ya, de la arbitrariedad o no de las divisiones administrativas romanas en la Península. Sabemos que una parte del territorio vettón, correspondiente a la zona de Ávila, queda adscrita a la citerior, tal y como transmite la noticia pliniana (Plinio, Nh. III, 19; ALBERTINI 1923, 108). En el caso concreto de la provincia de Toledo, los mencionados testimonios caesarobrigenses hacen suponer que el límite administrativo lusitano fue en origen y definitivamente establecido en algún lugar entre los territorios de Toletum y Caesarobriga.

Ptolomeo (II, 5, 7) cita a los Vettones como el pueblo más oriental de Lusitania, lo que también hace volver a un supuesto origen vettón de Caesarobriga, toda vez que ya sabemos de su adscripción a esta provincia. En cambio, entre las ciudades vettonas citadas por el autor no se encuentra ésta, aunque en algún caso se ha supuesto que la denominada Kottaeobriga pudiera ser identificada con una Caesarobriga situada en Ciudad Rodrigo, mientras otras interpretaciones la llevan a posiciones más occiden- tales (Almeida, Portugal) (2). Lo cierto es que la localización ptolemaica establece una misma posición meridiana para esta ciudad y para Augustobriga, lo cual hace pensar que, aún admitiendo un error de cálculo, la mencionada Kottaeobriga sería un enclave más oriental de lo que se ha supuesto.

Sin abandonar las fuentes clásicas, hay que añadir que Caesarobriga no está citada entre las mansiones de los itinerarios antiguos peninsulares. El Itinerario de Antonino menciona una vía que unía Emerita Augusta y Caesaraugusta (It. Ant. 438, 1-439, 4), a través de las mansiones de Lacipea, Leuciana, Augustobriga, Toletum, Titulcia, Conplutum (sic), Arriaca, Caesada, Segontia, Arcobriga, Aquae Bilbitanorum, Bilbili, Nertobriga y Segontia. La distancia entre mansiones en ningún caso es superior a XXX m.p. (entre Titulcia y Conplutum; It. Ant. 438,7 y 8 ), con la notable excepción del trayecto Augustobriga-Toletum, separadas por LV m.p. (3). Ahora bien, el recorrido de la vía en este tramo iba por el valle del Tajo; la ciudad de Caesarobriga tendría que encontrarse por fuerza en su camino, dada su situación intermedia entre Toletum y Augustobriga, y también como ellas, junto al Tajo; esto explicaría la excesiva distancia entre ambas mansiones, supuestamente correlativas y que no resultaría operativa para el viajero, máxime cuando sabemos de la existencia de un punto adecuado a mitad del recorrido, como es Caesarobriga, que es difícil suponer que no cumpliera una función de mansión para la que estaba perfectamente situada; es significativa, en este sentido, la presencia de Talavera como punto de descanso en los itinerarios de época moderna (Guía de Caminos 1748). Así pues, hay que suponer que su omisión en los itinerarios antiguos sea producto de un error.

Por otra parte, el apoyo arqueológico es fundamental. Entre los testimonios de la cultura material de los Vettones son significativos los hallazgos de toros y verracos, diseminados por diferentes poblaciones. Aún cuando ha quedado demostrado que este tipo de manifestaciones no son exclusivas de los Vettones, por los numerosos testimonios en terri-

(2) Sobre las diversas opiniones al respecto, vid. ROLDÁN 1968-69, 89, recogiendo la opinión de Cortés sobre la identificación con Ciudad Rodrigo, así como la que la situaba en Almeida, en Portugal (Ukert).

(3) It. Ant. 438, 6 y 7. Sobre este trayecto viario, ARIAS 1966, 278-280 y 1967, 373-374; ROLDÁN 1973, 86-87. 
torio lusitano-galaico, sí se considera que es propio de su cultura y, desde luego, en ningún caso se desarrolla entre los Carpetanos, por lo que constituye un claro elemento diferenciador. Ya Roldán (1968$69,104)$ había establecido la importancia de estas esculturas como elemento de separación, aunque en la fecha de publicación de su trabajo, los testimonios no eran tan abundantes y por tanto no permitían mayor precisión. G. López Monteagudo (1983b, 474-475) recoge todos los testimonios hallados hasta el momento de aparición de su obra, que para la provincia de Toledo se distribuyen de la siguiente manera: 1) verracos: Alcaudete de la Jara, Alcolea de Tajo, Calzada de Oropesa, Castillo de Bayuela, Las Herencias, Oropesa, Torralba de Oropesa, Torrecilla de la Jara y Totanes; 2) toros: Castillo de Bayuela, Talavera de la Reina y Totanes. Observando en el mapa (Figura 1) los lugares mencionados, se aprecia una línea diferenciadora, establecida, de sur a norte, por los hallazgos de: Torrecilla de la Jara, Alcaudete de la Jara, Las Herencias, Talavera de la Reina, Castillo de Bayuela, para enlazar con El Tiemblo, ya en provincia de Ávila. Al oeste de esta línea, siempre hablando de Toledo provincia, la proliferación de verracos está clara, con los mencionados de la zona de Oropesa y el de Alcolea de Tajo, presencia que también tiene continuidad, y con más intensidad, en las provincias de Ávila y Cáceres. En Toledo, sólo se da la excepción de Totanes, situado al suroeste de Toledo capital, y que, sin lugar a dudas, se integraría en Carpetania, por lo que se trataría de un hallazgo casual cuya explicación estaría lejos de cualquier participación carpetana, más bien en el asentamiento allí de elementos vettones, responsables de los dos testimonios.

Por lo que se refiere a la pieza de Talavera, fue publicada ya en 1958 por Jiménez de Gregorio, que da la noticia de la localización de esta escultura, o mejor, parte de ella, en la llamada Torre del Polvorín de la muralla (JIMÉNEZ DE GREGORIO 1958, 200-201, con noticias anteriores). Se trata de una pieza muy desgastada, que fue utilizada a modo de sillar, según opinión del autor, cuando se construyó la parte medieval de ladrillo.

Como hemos apuntado, los verracos no son considerados ya, a la luz de los hallazgos actuales, una manifestación exclusiva de la cultura vettona, pero sí son elemento característico de ella; de ahí la importancia de esta línea imaginaria que los hallazgos trazan de norte a sur por la parte oriental de la provincia de Ávila y aproximadamente central de la de Toledo, como última zona de control vettón antes de entrar en Carpetania, donde no se produce este fenómeno; Caesarobriga, a tenor de este tipo de hallazgos, quedaría englobada en la zona vettona.

En una ocasión anterior (GONZÁLEZ-CONDE 1987, 13ss.) ya establecimos un límite occidental para Carpetania, que, en la zona que nos ocupa, discurría entre Toletum y Caesarobriga, englobando en la región a lugares cuya situación y hallazgos arqueológicos y epigráficos parecían llevar a una identificación con los Carpetanos. Así ocurre con San Martín de Montalbán, Puebla de Montalbán, Escalonilla, Cármena, Maqueda, algunos de ellos claramente dentro del territorium de Toletum.

Por su parte, la documentación epigráfica aporta varios elementos importantes. En primer lugar, en lo que se refiere al aspecto religioso reflejado en las inscripciones, que permite introducir una nueva argumentación. El culto a las divinidades indígenas está documentado para la zona de Talavera. En los alrededores de la ciudad se halló la dedicación de L. Vibius Priscus a Togotus (CIL II 893; ALBERTOS 1983a, 486), divinidad escasamente atestiguada, que se ha querido identificar también con las menciones To... y Togot de otras inscripciones (4). También en Talavera se encontró la dedicación a Aricona (5), que María L. Albertos (1983a, 478) recogió como único testimonio de ese culto. Por fin, una dedicación a Ataecina procede de Caleruela (CORTÉS et alii 1984, n. ${ }^{\circ}$ 3), si bien es cierto que este lugar está más cercano a Augustobriga (Talavera la Vieja) que a Caesarobriga. Se trata de una inscripción dedicada por un eques del ala Vettonum, Bassus, a esta diosa, de la que hay varios testimonios que la circunscriben a un área entre el Tajo y el Guadalquivir, y más concretamente a los alrededores del Guadiana (BLÁZQUEZ 1975a, 39-42; BEAUJEU 1976, 439). El mayor número de epígrafes se integran en la región lusitana, con especial incidencia en Emerita Augusta, en donde su culto era oficial. Su nombre se recoge como Ataecina, Ataegina, o bien, frecuentemente, con el epíteto Turobrigensis (haciendo mención de la ciudad de

(4) To... (Ávila): CIL II 5861; BLÁZQUEZ 1975a, 173; RODRÍGUEZ ALMEIDA 1981, n. ${ }^{\circ} 44$ y 63 (explicando el problema de la inscripción). Togot (Botorrita, Zaragoza): TOVAR 1973, 374ss.; BLÁZQUEZ 1975a, 173 y 1979, 131ss.

(5) MORÁN C., AEA 17, 1944, 248, n. ${ }^{\circ}$ 18; Id. AEA 25, 1952, 157; BLÁZQUEZ 1975a, 30. 
origen de su culto), y también como Dea sancta Turobrigensis. La presencia de estos cultos indígenas en la zona contrasta con lo que habitualmente se encuentra en Carpetania, donde sólo conocemos la inscripción dedicada a Bandua (Sonseca) (6) como un caso aislado de culto indígena documentado entre los Carpetanos, quienes debieron asimilar las nuevas corrientes religiosas con bastante más facilidad que sus vecinos Vettones.

La coincidencia de dos dedicaciones a dioses indígenas en la propia Caesarobriga (la primera de ellas a pocos $\mathrm{kms}$. de la ciudad) es claro indicio de esta resistencia a la integración en la cultura romana entre los Vettones. Junto a ellos, el caso de Caleruela es testimonio de una continuidad cultural desde la zona de Augustobriga a la de Caesarobriga, así como de su identidad con el resto del territorio vettón. Hacia oriente, dejando aparte el mencionado caso de Sonseca, todos los testimonios religiosos que se han conservado son relativos a dioses romanos, en claro contraste con los hallazgos de Caesarobriga, que la relacionan más con el mundo vettón.

Volviendo a la inscripción de Bassus, el dedicante de la diosa Ataecina, puede decirse que es un eques del ala Vettonum, como él mismo se encarga de hacer constar en el epígrafe. Aunque el lugar del hallazgo se integre más bien en el territorium de Augustobriga, sirve para dejar constancia de que esa zona entre esta ciudad y Caesarobriga se encuentra en pleno dominio vettón.

El ala Vettonum c.R. está atestiguada en Britannia (Aquae Sulis) en época flavia, por el epitafio de L. Vittelius Tancinus (7), siendo ésta la primera guarnición conocida para la unidad. También hay otro testimonio en Fenni Fach (Britannia) (ROLDÁN 1974, n. ${ }^{\circ}$ 104; ALBERTOS 1979, n. ${ }^{\circ} 9$ ). La condición ciudadana fue otorgada a la unidad, de forma honorífica, en época flavia (LE ROUX 1982, 94 n. 78), y figura ya en los testimonios de Britannia; el hecho de que el individuo de Caleruela no lo haga constar, parece indicar que su dedicación fue hecha antes de dicha concesión, durante la estancia del ala en la Península, desde donde posteriormente saldría hacia Britannia. La utilización de los Vettones en la caballería romana supone la destreza

(6) FITA 1892, 146ss.; BLÁZQUEZ 1975a, 47 y 1983, 296.

(7) CIL VII 52; ILS 2517; ROLDÁN 1974, n. ${ }^{\circ}$ 103; ALBERTOS 1979, n. ${ }^{\circ} 8$; LE ROUX 1982, n. ${ }^{\circ} 81$. de éstos como jinetes, cosa que no es de extrañar en una zona en donde los caballos, y la ganadería en general, son elemento importante. Auque sin aludir a los Vettones directamente, las fuentes clásicas transmiten noticias de este tipo para los Lusitanos y Celtíberos; así, aparte de menciones generales a la abundancia de caballos en Hispania (Plinio, $N h$. XXXVII, 203; Mela, Chor. II, 86; y muchas otras a la ganadería), hay que añadir el conocido mito sobre las fértiles yeguas lusitanas, fecundadas por el viento, así como muchas otras referencias a los caballos en Bética y Celtiberia (BLÁZQUEZ 1975b, 126-129, con una recopilación de las fuentes). Por el contrario, el caballo no parece tener esa importancia en Carpetania, para la que no tenemos conocimiento de menciones en los autores clásicos, quizá debido a que no fuera tan abundante como entre los otros pueblos de la Meseta sur, Lusitania y Bética. Por otra parte, no hay ningún testimonio que haga pensar que los Carpetanos formasen una unidad auxiliar en el ejército romano, no ya como jinetes, sino siquiera en una cohors; quizá esto se deba a una falta de condiciones y preparación para la lucha, lo cual, por otra parte, explicaría su aparente pasividad durante la conquista romana de la Meseta. En cualquier caso, éste es un aspecto que les separa de los Vettones.

Desde el punto de vista onomástico, resulta más difícil establecer una línea clara entre Vettones y Carpetanos. A la luz de las inscripciones, son muchos los nombres indígenas que se repiten en ambas regiones, por lo' que ya María L. Albertos (1983b, 869ss.) afirmó que no podían separarse en dos grupos absolutamente diferenciados. En Caesarobriga la onomástica conocida responde a esa tónica general de ambas zonas, ya que la mayor parte de los nombres indígenas de la ciudad y sus alrededores no son exclusivos de Vettones ni tampoco la relacionan o separan de Carpetania, excepto por lo que se refiere a un determinado grupo onomástico, representado en Talavera de la Reina por tres inscripciones. Se trata de $C$. Magius Ton[g]ius (8); Tanginus, en el epitafio de su hija Annia (CIL II 903); y Tongetamus, en su propia inscripción funeraria (CIL II 5334). Dentro del grupo, los testimonios de Tancinus-Tanginus corresponden casi en su totalidad a la provincia acutal de Cáce-

(8) CIL II 916 (quizá Ton/clius), que por error fígura como procedente de Toledo en GONZÁLEZ-CONDE 1987, 87, n. 53. 


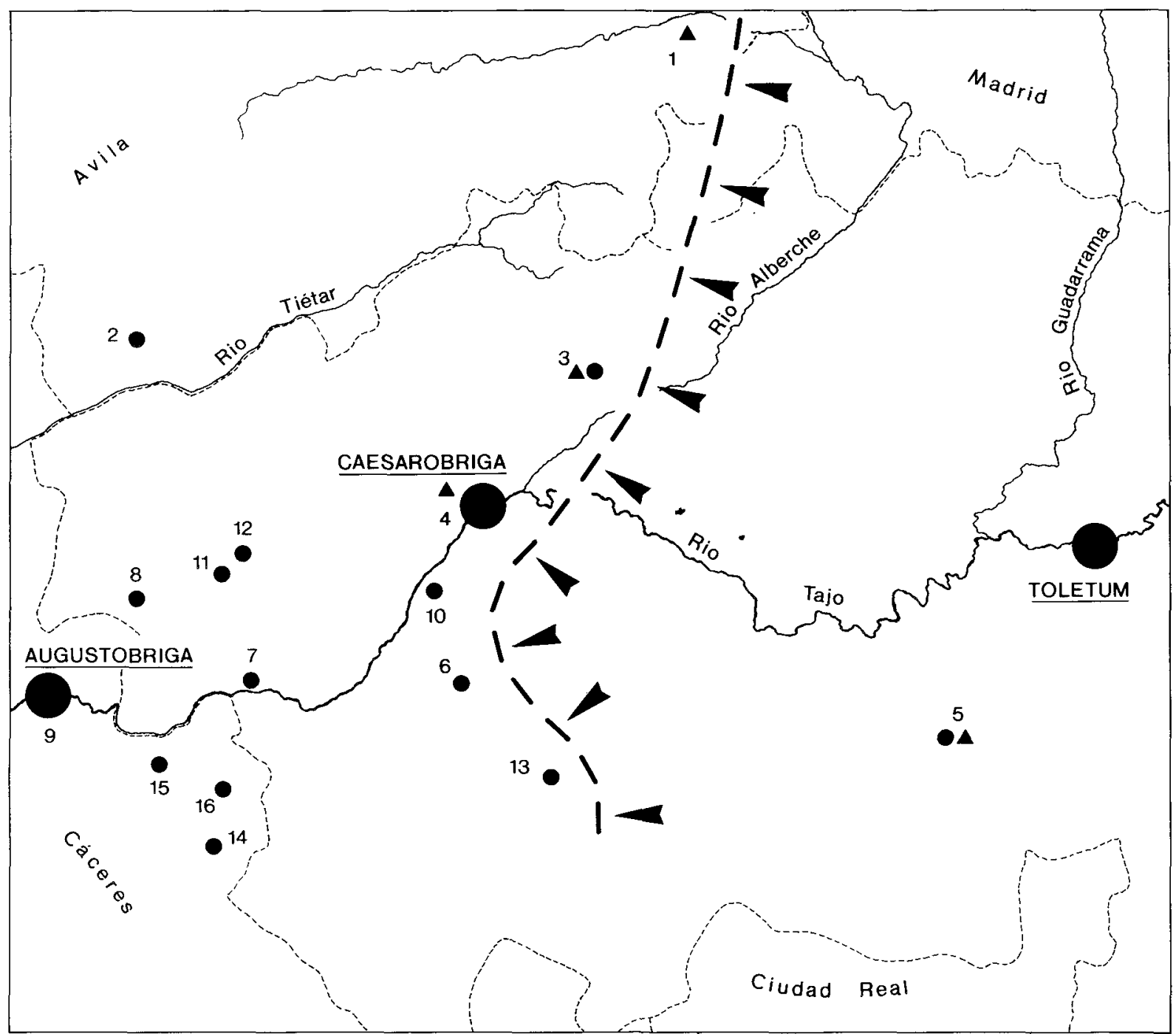

Figura 1.-Dispersión de los hallazgos de toros y verracos en la zona occidental de la provincia de Toledo, con indicación de una línea imaginaria que limitaría el área de influencia vettona. Los triángulos indican los hallazgos de toros, mientras que los círculos señalan los de verracos. Leyenda: 1.- El Tiemblo; 2.- Candeleda; 3.- Castillo de Bayuela; 4.- Talavera de la Reina; 5.- Totanes; 6.- Alcaudete de la Jara; 7.- Alcolea del Tajo; 8.- Calzada de Oropesa; 9.- Talavera la Vieja; 10.- Las Herencias; 11.- Oropesa; 12.- Torralba de Oropesa; 13.- Torrecilla de Ia Jara; 14.- Carrascalejo de la Jara; 15.- Valdelacasa de Tajo; 16. - Villar del Pedroso.

res, con algún caso en Lusitania, mientras que las variantes Toncius, Tongius, Toncetamus, Tongeta, Tongatus, también son propias del área lusitanovettona, con algún caso en la zona astur-galaica (9).

En fin, todo apunta hacia la idea de que la zona de la provincia de Toledo que corresponde a Cae-

(9) PALOMAR LAPESA 1957, 124, 130-31, 134, 144; ALBERTOS 1966, 135, 229, 230, 290, 295 y 1979, 48; Id. 1983b, 870ss. sarobriga y sus alrededores se integraba dentro del mundo vettón; así se deduce del estudio conjunto de las fuentes literarias, arqueológicas y epigráficas, que establecen diferencias claras con los Carpetanos. Caesarobriga sería el municipio romano más oriental de los establecidos en territorio vettón, aunque su situación probablemente hacía que fuese una zona en contacto con Carpetania, especialmente teniendo en cuenta su forzosa localización en el trayecto de la vía que venía de Toletum y se dirigía por Augustobriga a Emerita Augusta, sin olvidar ade- 
más que los factores geográficos también favorecían los contactos entre estas ciudades situadas a lo largo de la ribera del Tajo. En este aspecto del trazado viario, la omisión del Itinerario de Antonino ha de ser forzosamente un error, en modo alguno achacable al desconocimiento general de esa ciudad, que no careció de importancia. Caesarobriga alcanzó la condición de municipio, como demuestran las inscripciones de la citada flaminica municipal y provincial (CIL II 895), Domitia Proculina, y la de un individuo que ocupó allí todas las magistraturas ( $L$. Annius Placidus, CIL II 896), produciéndose este cambio de status en una fecha que ya ha sido establecida en época flavia (WIEGELS 1985, 74), dato que queda reforzado por la adscripción de la ciudad a la tribu Quirina (CIL II 896; CIL II 5320, de un personaje cuyo nombre es dudoso; CIL II 913, de C. Licinius Fuscinus).

El municipio contaba con una élite social importante, quizá desde la concesión del privilegio o incluso antes, relacionada por lazos de parentesco que les permitirían cierto acaparamiento de las magistraturas. Conocemos a L. Annius Placidus, que fue aedilis, quaestor y Ilvir en su ciudad natal; es decir, desarrolló el cursus más completo que la administración municipal permitía, hasta el grado más alto (FRANCISCO MARTÍN 1977, n. ${ }^{\circ} 20$ ). Su mujer, Domitia Attia, que le dedica el epitafio (CIL II 896; también el suyo propio en CIL II 897) puede estar emparentada con la mencionada flaminica, por la similitud del nomen, teniendo el cognomen de esta última a su vez una posible relación con el de $A m m i a$ Procula (JIMÉNEZ DE GREGORIO 1969, 212, Fig. 13), también de Talavera. Además, esta mujer que ocupó el flaminado en su propio municipio, debió ser uno de los personajes más influyentes del mismo, tanto por la concesión del título honorífico de flaminica perpetua, como por su ascenso al flaminado provincial (ETIENNE 1974r., 166, 167, 239).

El desarrollo institucional y social de Caesarobriga debió tener un impulso económico que convirtiera a la ciudad en un lugar con la importancia necesaria para acceder al rango de municipio, con una élite fuertemente apoyada sobre la base de un potencial económico. Entre las actividades que más influyeran en este desarrollo pudo estar la industria cerámica, por la existencia allí de alfares de los que ha quedado constancia (JUAN TOVAR 1983, 16375), y que permitirían a la ciudad repetir un proceso parecido al ya conocido para otros municipios
(ESPINOSA y PÉREZ RODRÍGUEZ 1982, pas$\operatorname{sim}$ ), en donde ese tipo de actividades actuaron como motor económico para el desarrollo municipal.

Para concluir, y ante la imposibilidad de una mayor precisión, parece que la cuestión de la delimitación entre Vettones y Carpetanos en la actual provincia de Toledo permite el establecimiento de una línea aproximada que, pasando entre Toletum y Caesarobriga, discurriría con dirección norte-sur, dejando a occidente los testimonios de verracos en esta provincia hasta enlazar con la de Ávila. Hacia el este, se entraría ya en el dominio de los Carpetanos, mientras al oeste quedaba el mundo vettón, dentro del cual se integraría plenamente el territorio de Caesarobriga y su zona de influencia.

\section{BIBLIOGRAFÍA}

ALBERTINI, E. 1923: Les divisions administratives de l'Espagne romaine. París.

ALBERTOS Firmat, M. ${ }^{a}$ L. 1966: La onomástica personal primitiva de Hispania. Tarraconense y Bética. Salamanca.

- 1979: «Vettones y Lusitanos en los ejércitos imperiales«, en Homenaje a C. Callejo Serrano, Cáceres, 31 ss.

- 1983a: «Teónimos hispanos»; Apéndice en J. M. Blázquez, Primitivas religiones ibéricas II, Religiones prerromanas. Madrid, 477ss.

- 1983b: «Onomastique personelle indigène de la Pèninsule lbèrique sous la domination romaine», en $A N R W$ II, 29.2, Berlín, 853ss.

ARIAS Bonet, G. 1966: «El Itinerario de Antonino y los grandes ríos», en Miliario Extravagante 11, 278ss.

- 1967: Augustobriga vettona, en Miliario Extravagante 13, 373-374.

BEAUJEU, J. 1974: «Cultes locaux et cultes d'Empire dans les provinces d'Occident aux trois prèmiers siècles de notre Ere«, en Assimilation et résistance à la culture grécoromaine dans le monde ancien. Travaux du VII ${ }^{\mathrm{e}}$ Congrés Int. d'Etudes Classiques (Madrid), Bucarest-París, 433ss.

BLÁZQUEZ, J. M. ${ }^{\text {a }}$ 1975a: Diccionario de la religiones prerromanas de Hispania. Madrid.

- 1975b: La Romanización. Vol II. Madrid.

- 1979: «Últimas aportaciones a las religiones primitivas de Hispania», en Homenaje a C. Callejo Serrano, Cáceres, 131 ss.

- 1983: Primitivas religiones ibéricas II. Religiones prerromanas. Madrid.

CORTÉS, S. et alii 1984: «Nuevas inscripciones romanas del Museo de Santa Cruz de Toledo», en Museos 3, 73ss.

ESPINOSA, U. y PÉREZ RODRÍGUEZ, A. 1982: «Tritium Magallum; de ciudad peregrina a municipio romano», en $A E A$ 55, 65ss.

ETIENNE, R. 1974r: Le culte imperial dans la Péninsule Ibérique d'Auguste à Diocletien. París (ed. original 1958).

FITA, F. 1892: «Antigüedades romanas», en $B R A H$ XX1, 146ss. 
FRANCISCO MARTÍN, J. 1977: «Los magistrados municipales de Lusitania durante el Alto Imperio», en Memorias de Historia Antigua I, 227ss.

GONZÁLEZ-CONDE, M. ${ }^{a}$ P. 1987: Romanidad e Indigenismo en Carpetania. Alicante.

GUÍA... 1748: Guía de Caminos, en Nuevo estilo y formulario de escribir cartas missivas. Madrid.

JIMÉNEZ DE GREGORIO, F. 1958: «Hallazgos arqueológicos en La Jara VIII», en $A E A$ XXXI, n. ${ }^{\circ} 97$ y 98,199 ss.

- 1969: «Hallazgos arqueológicos en la provincia de Toledo», en $A E A$ XLII, n. ${ }^{\circ} 119-120$.

JUAN TOVAR, C. 1983: «Elementos de alfar de sigillata hispánica en Talavera de la Reina (Toledo)», en Boletín del Museo Arqueológico Nacional II, 163-175.

LE ROUX, P. 1982: L'armée romaine et l'organisation des provinces ibériques d'Auguste à l'invasion de 409. París.

LÓPEZ MONTEAGUDO, G. 1983a: Expansión de los verracos y características de su cultura. Madrid.

- 1983b: «Localidades donde han aparecido verracos»; Apéndice en J. M. ${ }^{a}$ Blázquez 1983.
MARTÍN VALLS, R. 1974: Protohistoria y romanización de los Vettones. Valladolid.

PALOMAR LAPESA, M. 1957: La onomástica personal prelatina de la antigua Lusitania. Salamanca.

RODRÍGUEZ ALMEIDA, E. 1981: Ávila romana. Ávila.

ROLDÁN HERVÁS, J. M. 1968-69: «Fuentes antiguas para el estudio de los Vettones», en Zephyrus XIX-XX, 73ss.

- 1973: Itineraria Hispana. Valladolid.

- 1974: Hispania y el ejército romano. Salamanca.

SALINAS DE FRÍAS, M. 1982: La organización tribal de los Vettones. Salamanca.

TOVAR, A. 1973: «Las inscripciones de Botorrita y de Peñalba de Villastar y los límites orientales de los Celtiberos», en $H A$ III, 367ss.

VALIENTE, S. y BALMASEDA, L. 1983: «Hacia una delimitación de la Carpetania en la edad del Hierro II», en Homenaje al Prof. M. Almagro Basch I, Madrid, 135ss.

WIEGELS, R. 1985: Die Tribusinschriften des römischen Hispanien. Ein Katalog. Berlín. 